\title{
Verflüssigende (Un-) Sicherheiten. Über Räumlichkeiten des Strassenhandels am Beispiel Brasiliens
}

\author{
Marc Redepenning, Jena, Henriette Neef, Leipzig, \\ Edvânia Torres Aguiar Gomes, Recife
}

\section{Sicherheit, Unsicherheit und «Raum»}

Fragen nach Sicherheit und Unsicherheit haben in den letzten Jahren ein erhöhtes Interesse nach sich gezogen. Man geht wie selbstverständlich davon aus, dass das gesellschaftliche Dasein objektiv risikoreicher geworden ist (BECK 1986: 31ff.).

Daneben hat sich eine andere Sichtweise konsolidiert, die bemerkt, dass es sich bei der zunehmenden Behandlung von Problemen unter den Aspekten von Sicherheit und Unsicherheit um die Durchsetzung eines Denkstils handelt: " $(\mathrm{N}) \mathrm{o}$ issue is essentially a security problem. (In)security is not an objective condition» (BALzACQ 2005: 177). Man kennzeichnet gesellschaftliche Erscheinungen somit als sicher bzw. unsicher und kann, darauf basierend, das Risiko kalkulieren, das man eingeht, wenn man nichts für die Sicherheit und somit gegen die Unsicherheit unternimmt.

Dazu bedarf es aber erstens Vorstellungen, «was in der sozialen Kommunikation unwidersprochen als sicher behandelt wird» (LUHMANN 2003: 28). Und es bedarf zweitens der Identifizierung von Unsicherheiten, um durch ihre Verminderung ein Mehr an Sicherheit zu erzeugen. Im Rahmen einer solchen Orientierung widmen wir uns demnach der Unterscheidung von Sicherheit und Unsicherheit und nehmen einen relationalen Blickwinkel ein. Die Unterscheidung Sicherheit/Unsicherheit bildet ein gesellschaftliches Beobachtungsraster. So kann man schauen, welche Seite dieser Unterscheidung von Beobachtern (beispielsweise politischen Organisationen oder lokalen Wirtschaftsverbänden) genauer bestimmt wird. Es sei betont, dass jeder Beobachter eine andere Vorstellung von Sicherheit hat. In Bezug auf den Strassenhandel, der im Folgenden auf seine Sicherheiten wie Unsicherheiten diskutiert wird, lässt sich beispielsweise eine Vielzahl von unterschiedlichen Bestimmungen über seine Sicherheit (etwa in Form sozialer Netzwerke, einer flexiblen Grundversorgung der Bevölkerung sowie einer flexiblen Einkommensgenerierung für die Händler) oder über seine Unsicherheit (etwa als Blockieren des Verkehrs, Attraktion für Taschendiebe, Platzverbote für die Strassenhändler) finden (Bromley 2000).
Prozesse des Beobachtens von Sicherheit und Unsicherheit stehen am Anfang eines analytischen Vorgehens, das klären will, was in der Gesellschaft als sicher und was als unsicher thematisiert wird (vgl. BuzAN, WæVER \& De Wilde 1998; Buzan \& WÆVER 2009). Welche natürlichen und gesellschaftlichen Phänomene werden als Bedrohung für die Sicherheit eines sozialen Kollektivs angesehen? Die Themen, die unter dem Aspekt der Versicherheitlichung betrachtet werden, sind zahlreich und umfassen beispielsweise den öffentlichen Raum, die Bewegung von Gütern und Waren im lokalen oder globalen Verkehrsraum, das Individuum oder, wie hier diskutiert, das soziale System der Strassenhändler (camelôs).

Bei der Herstellung gesellschaftlicher Sicherheit spielen metrisch-räumliche Figurationen im Sinne der Relationierung physisch-materieller Objekte eine zentrale Rolle. Dieses räumliche Ordnen der Dinge soll die Sicherheit an einem konkreten Ort gewährleisten bzw. erhöhen. Raum kann als eine unter zahlreichen «techniques of government/governance for framing (in)security» (STRITZEL 2007: 376) verstanden werden, die besonders innerhalb einer politischen Rationalität der «ordnenden Trennung» von hoher Bedeutsamkeit ist. Eine ordnende Trennung äussert sich in Praktiken raumbezogener Segmentierung, wenn es darum geht, als unsicher taxierten Personen «ihren Platz zuzuweisen». Erkennbar wird dies etwa in jüngeren stadtentwicklungspolitischen Aktivitäten zur Einhegung und Sesshaftmachung von Strassenhändlern in sogenannten Shopping Centers des informellen Einzelhandels (brasilianisch: camelôdromos oder shopping populares). In den Bewegungen der Strassenhändler wird häufig ein Moment von Unsicherheit gesehen (s.u.), weil sie sich einer allgemeinen Planbarkeit, Eindeutigkeit und damit auch Kontrolle entziehen. Aus Sicht der Strassenhändler liegt jedoch in ebendiesen Bewegungen eine soziale und ökonomische Sicherheit, da durch sie soziale Netzwerke reproduziert und ein standortvariables Einkommen ermöglicht werden.

Das Anliegen des Artikels ist nun, exemplarisch und mit regionalem Bezug $\mathrm{zu}$ Brasilien die räumlichen Komplexitäten hinsichtlich Fragen der Sicherheit und Unsicherheit am Beispiel des politischen Umgangs mit dem Strassenhandel zu verdeutlichen. Es wird hervorgehoben, dass Raumstrategien der ordnenden Trennung auf einem metrischen und festen Raumverständnis beruhen, das die spezifischen sozio-kulturellen Räumlichkeiten des Strassenhandels nur unzurei- 
chend abbildet. Diese Räumlichkeiten werden unter Zuhilfenahme des Konzeptes des flüssigen Raumes (Mol \& Law 1994) genauer erörtert. Mit Bezug zu den übergeordneten Fragen der Unterscheidung Sicherheit/Unsicherheit wird schliesslich auf problematische Implikationen einer Engführung des Raumbegriffs als Relationierung physisch-materieller Objekte bzw. als fester und geometrischer (Container-) Raum hingewiesen. Dementsprechend setzt der Artikel einen Schwerpunkt auf die Analyse unterschiedlicher, oftmals auch kontradiktorischer Räumlichkeiten, wie sie an den Bewegungen des Strassenhandels sowie den Massnahmen seiner Regulation beobachtet werden können. Wir verstehen dabei «Bewegung» nicht ausschliesslich in einem metrisch-räumlichen Sinne, etwa als (zirkuläre) räumliche Mobilität. Die Konzentration des Artikels auf die Räumlichkeiten des Strassenhandels bedeutet ferner, dessen ökonomische und soziale Aspekte nur randlich und keineswegs erschöpfend zu thematisieren.

\section{2 (Un-) Sicherheit, Raum und Stadt}

In diesem Text behandeln wir Sicherheit und Unsicherheit als eine Unterscheidung, die in unterschiedlichen zeitlichen und räumlichen Kontexten auch unterschiedliche Bedeutungen erfährt. In der Konsequenz macht es dann wenig Sinn zu behaupten, dass ein Ort per se sicher oder unsicher ist. So kann nachts die Anwesenheit von mehreren Personen, wie z.B. an Bushaltestellen, durchaus als Element der Sicherheit empfunden werden. Tagsüber kann hingegen eine hohe Dichte von Personen am gleichen Ort als Bedrohung oder Element der Unsicherheit wahrgenommen werden.

Zur Bezeichnung jener Beobachtungen urbaner Phänomene unter der Leitdifferenz von Sicherheit/Unsicherheit schlagen OsBorne und Rose (1999:753ff.) den Begriff der risky city vor. Er umfasst unter anderem Praktiken und Technologien, mit denen urbane Nachbarschaften sicherer gemacht werden sollen. Materielle wie immaterielle Kräfte sollen gelenkt werden, um eine bestimmte Ordnung in Bezug auf die Praktiken der Bevölkerung zu implementieren (OsBorne \& Rose 1999: 738). In ihrer Analyse der risky city thematisieren OSBORNe und Rose u.a. auch raumbezogene Strategien, die den Umgang mit den Unsicherheiten der modernen Stadt dirigieren sollen. Diese Strategien äussern sich zunächst in einem permanenten raumbezogenen Monitoring der Unsicherheit. Darauf aufbauend lassen sich dann raumbezogene Strategien der Verminderung oder doch zumindest der Zähmung der als unsicher beobachteten Phänomene entwickeln: Hierzu gehören die Überwachung von Gebieten mittels Videokameras, aber auch die Politik der Zonierung der Stadt, um über Abgrenzungen und Zuweisungen eine Kontrolle der als unsicher erachteten Elemente anzustreben. Das Spektrum der Zonierungen ist breit: es reicht von Standortgenehmigungen für Prostituierte und Strassenhändler bis zur Fixierung von unsicheren Elementen in modernen Gefängnissen. Auch die hier besprochenen Shopping-Center des informellen Einzelhandels (camelôdromos), also Einrichtungen, in die Strassenhändler (camelôs) umgesiedelt werden, können als Teil einer Politik der Zonierung identifiziert werden.

Die folgenden Abschnitte des Textes werden sich genauer mit solchen raumbezogenen Lösungen von Problemen der Unsicherheit bzw. der mangelnden Sicherheit beschäftigen. Gleichwohl erkennen wir an, dass dies nur einen Teil der Strategien des Umgangs mit urbaner Sicherheit und Unsicherheit repräsentiert (Strategien, die an der Verhaltenssteuerung des Individuums ansetzen, wären hier beispielsweise zusätzlich zu nennen).

\section{Die politische Regulation des Strassenhandels}

Bei der Betrachtung jüngerer Stadtentwicklungsprogramme und ihrer öffentlichen Diskussion in brasilianischen Städten fällt auf, dass diese stark von Semantiken wie Sicherheit, Ordnung und Sauberkeit geprägt sind - in diesem Sinne passen sie sich in die Rationalität der risky city ein (Dos ReIs 2003: 45ff; PereIra 2007). Es handelt sich primär um Programme, die eine Revitalisierung von Stadtzentren vorsehen. In den Programmen werden die Stadtzentren zunächst als unsaubere und unsichere Orte deklariert, während ihre Wiederbelebung nur in Form sauberer und sicherer Räume denkbar ist. Massnahmen der Restaurierung von Gebäuden, der Instandsetzung von Strassenzügen sowie der Gestaltung von Grünflächen sollen diese Gebiete wieder zum ästhetischen Anziehungspunkt für Konsum, Freizeit und Tourismus machen. Zeitgleich gilt es, die betroffenen Gebiete zunächst von den verunreinigenden und verunsichernden Elementen zu befreien. In der politischen Praxis betreffen diese Regelungen insbesondere marginalisierte Akteure wie Obdachlose, Bettler, Strassenkinder sowie den Komplex des Strassenhandels. Der Strassenhandel, der diese Gebiete häufig seit Jahrzehnten bevölkert, wird als Element der Unsicherheit thematisiert. Er stellt eine gravierende Bedrohung dieses neuen Raumes dar.

Daher finden sich Strassenhändler vermehrt vor ernsthafte Probleme gestellt:

«(T)hey are perceived not as agents of innovation, but as anathema to city marketers who claim traders congest streets and create broken windows> that generate disorder, blight and crime» (Donavan 2008: 30). 


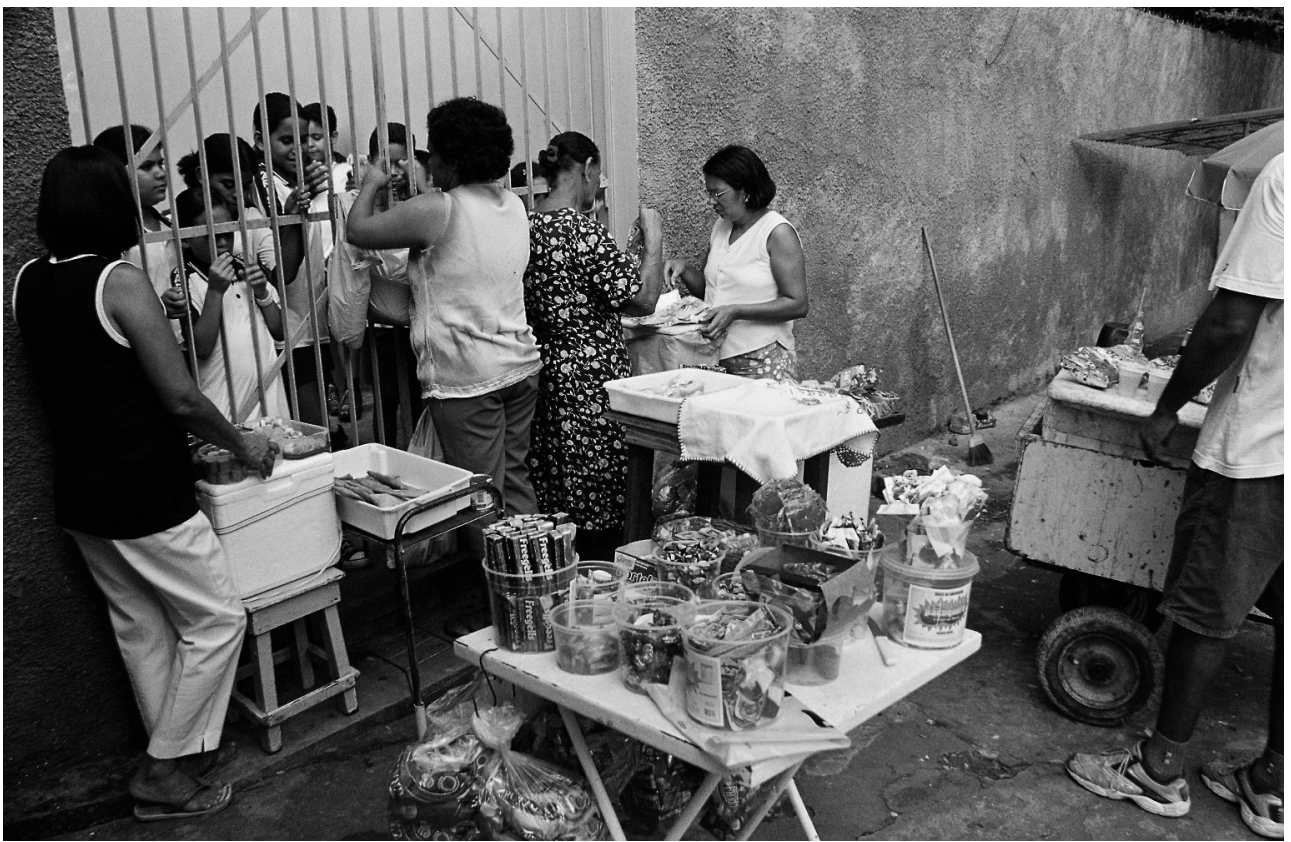

Abb. 1: Spontane Ansammlung von Strassenhändlern vor einer Schule in Recife Spontaneous grouping of street traders in front of a school in Recife Regroupement spontané de vendeurs de rue devant une école de Recife Foto: H. NeEF (2006)

Der Strassenhandel wird als verunsichernde, weil nicht klar taxierbare Form begriffen, die oftmals spontan und zeitlich begrenzt an bestimmten Orten auftaucht (Costa 1989). Die brasilianische Architektin Costa spricht in diesem Zusammenhang auch von Blasenräumen (espaços de bolha), die durch die Ansammlungen von Strassenhändlern an städtischen Knotenpunkten erzeugt werden. Zumeist handelt es sich hierbei um Einrichtungen der technischen oder sozialen Infrastruktur, die zu bestimmten Tageszeiten stark frequentiert werden (z.B. Ampeln, Haltestellen, Parkanlagen, Strände, öffentliche Einrichtungen). Aber auch besondere Ereignisse wie Konzerte, Fussballspiele, Staus oder eine Schulpause (Abb. 1) sind Anlass, dass Strassenhändler sich spontan sammeln und temporäre Verkaufsräume errichten. Der Begriff des Blasenraumes erlaubt, das Kommen und Gehen vieler Strassenhändler als rhythmisch wiederkehrenden metrisch-relationalen Raum zu beschreiben, da konkrete Stellen im städtischen Raum in der Regel iterativ und zeitlich begrenzt besetzt werden. Dieses Verhältnis von temporärer Deterritorialisierung und Reterritorialisierung, und damit die Herstellung von flexiblen Territorien (Haesbaert 2004; Souza 1995), kann sich sowohl auf die gleiche Stelle wie auf verschiedene Stellen des urbanen Raumes beziehen.

Das führt dazu, dass den Strassenhändlern jene Attribute des Verlässlichen, des Rechtmässigen und Berechenbaren fehlen, die in der Rationalität der risky city mit Sicherheit und Ordnung verbunden werden. Im Gegenzug verkörpern sie viel eher das Chaotische, das Nicht-Berechenbare und somit Unsichere. So ergaben beispielsweise Umfragen in Porto Alegre, dass die Mehrheit der Bevölkerung den Strassenhandel aufgrund dieser Attribute in den betroffenen Gebieten als «fehl am Platz» empfindet (Meucci Nique 2006: 84f.).

Dieses negative Bild spiegelt sich in zahlreichen, in den letzten Jahren instituierten stadtentwicklungspolitischen Massnahmen wider, die das Ziel haben, den Strassenhandel zu zähmen und ihn an einem Ort zu verankern (s.u. am Beispiel der camelôdromos). Verschiedene Autoren erkennen in diesen Massnahmen eine purification of space (SIBLEY 1988) oder auch eine Intervention im Namen urbaner Hygiene, mit dem Ziel, eine aseptische räumliche Ordnung herzustel- 


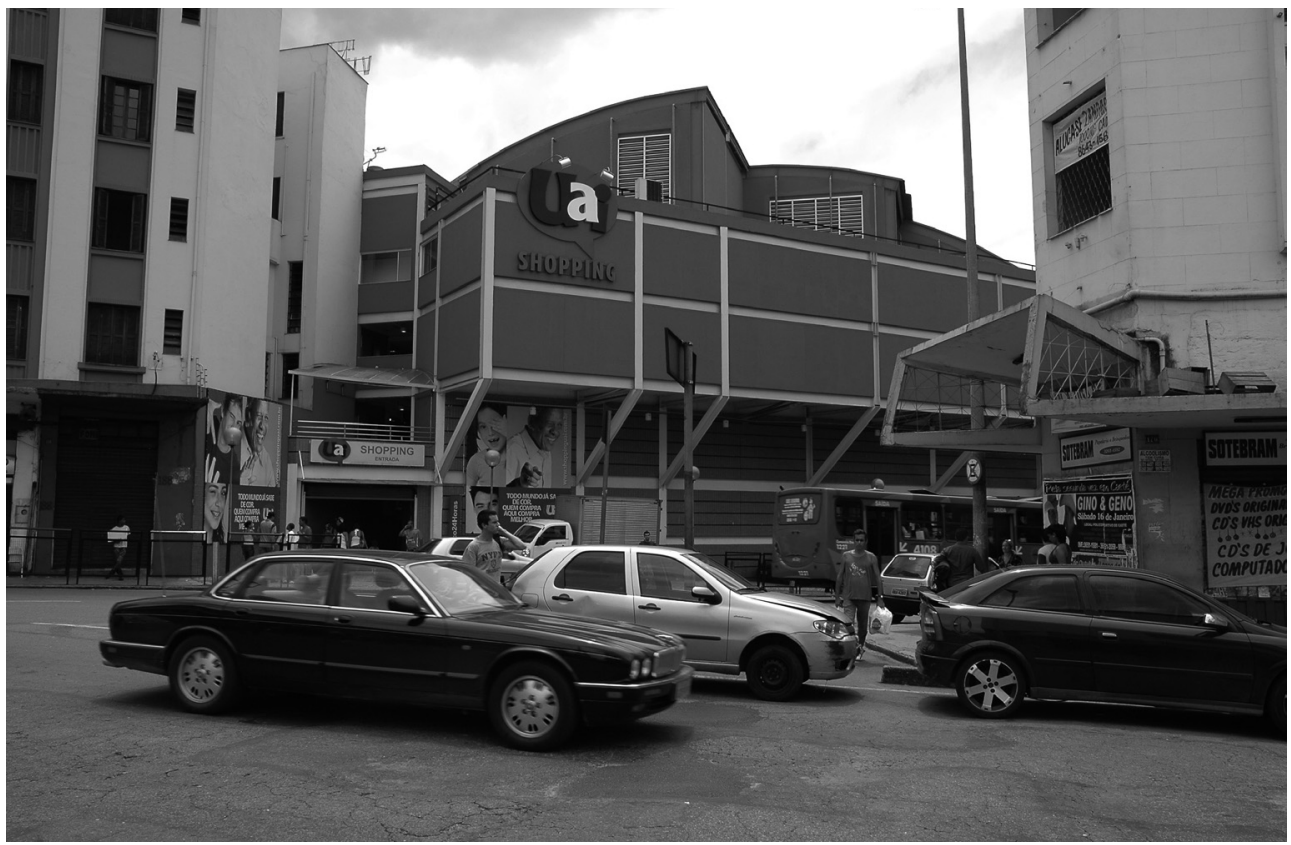

Abb. 2: Camelôdromo (Uai Shopping) in Belo Horizonte

Camelôdromo (Uai Shopping) in Belo Horizonte Camelôdromo (Uai Shopping) à Belo Horizonte

Foto: H. NeEF (2010)

len (Dos ReIs 2003; Januzzi 2005). Die dislozierten Händler stellen so keine Gefahr für die neue Ästhetik der Innenstadt als Voraussetzung eines ungestörten und damit «sicheren» touristischen Erlebnisses dar (Middleton 2003). Im Vordergrund steht die (Wieder-) Herstellung eines Stadtbildes, das einer unbeweglichen Postkartenidylle gleicht (Pereira 2007). Hervorzuheben ist, dass bei diesen politischen Massnahmen nicht die Abschaffung der Komponente der Informalität, die mit dem Strassenhandel einhergeht, zentral ist. Vielmehr handelt es sich um Versuche der Zähmung der Nichtsteuerbarkeit des Strassenhandels, weil auf die räumlichen Bewegungen und chaotisch anmutenden Konstellationen der Strassenhändler ein diffus vorhandenes Gefühl der Unsicherheit projiziert wird.

Wie dieser als unsicher erachtete Strassenhandel durch raumbezogene Organisation zu regulieren versucht wird, kann man beispielsweise am Aufkommen von camelôdromos sehen - Phänomene, die jüngst in vielen Städten Brasiliens zu beobachten sind (Abb. 2). Vormals mobile Strassenhändler können nun ihre Waren in diesen Shopping Centers des kontrollierten informellen Handels anbieten. Hierfür stehen ihnen kleine abgetrennte Boxen - also Raumcontainer - zur Verfügung, die sie monatlich anmieten und nach festgelegten Öffnungszeiten bewirtschaften sollen (Abb. 3). In São Paulo bemühten sich bereits ab den 1990er Jahren Interessensverbände um die Begründung der angeblichen Notwendigkeit der camelôdromos. Ihre Argumente sind durch die Inanspruchnahme des Beobachtungsrasters von Sicherheit/Unsicherheit bestimmt:

«Der informelle Handel okkupiert öffentliche Räume ohne jegliche Organisation oder irgendein Kriterium. Dadurch stimuliert er die Unsicherheit der Personen und des Kulturerbes der Innenstadt, vor allem dort, wo er besonders dicht gedrängt ist. Der Fussgänger ist der erste Geschädigte durch die Verstopfung der Fusswege» (Associação VIVA o Centro 1994: 13, Übersetzung der Autoren).

«Die öffentliche Sicherheit leidet unter dem camelô. Es ist bekannt, dass viele Stände als Versteck dienen und Beobachtungspunkte für die Marginalen sind» (ebd., Übersetzung der Autoren). 


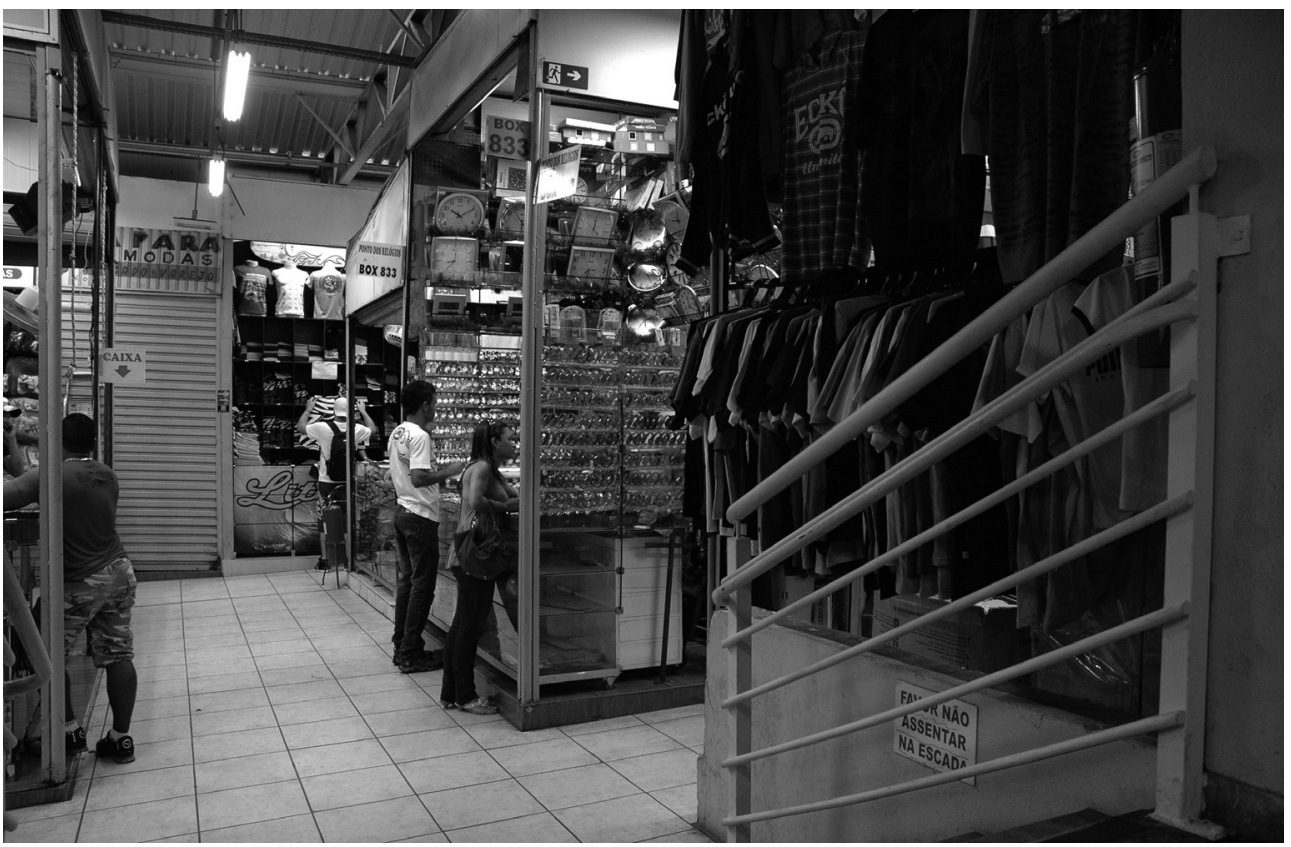

Abb. 3: Verkaufsboxen in einem camelôdromo (Shopping Oiapoque), Belo Horizonte

Shop containers in a camelôdromo (Shopping Oiapoque) in Belo Horizonte

Points de vente dans un camelôdromo (Shopping Oiapoque), Belo Horizonte

Foto: H. Neef (2010)

Die Aussagen betonen die durch die Bewegung hervorgerufenen Ballungen der Strassenhändler, wie es auch Donavan hervorhebt:

«Government officials typically restrict vending on the grounds that street traders increase pedestrian and vehicular congestion, cause traffic accidents and impede the flow of emergency vehicles» (Donavan 2008: 33).

Hinter den camelôdromos steht demnach die Idee, über Planungen und Regelungen die Strassenhändler an einem geeigneten Ort einzuhegen und sie somit einer anderen räumlichen Ordnung zu unterwerfen - einer Ordnung des starren und festen Raumes (Dos Reis 2003; Dos Santos 2001). Die Organisation dieser Räume folgt Richtlinien hinsichtlich der Hygiene, der Ästhetik und Sicherheit (CARDoso \& MarTINS 2005). Camelôdromos können somit als Teil einer Logik identifiziert werden, die versucht, eine neue urbane Ordnung über die Ausweisung von bestimmten Aktivitätszonen und die Einhegung von Händlern zu etablieren. An diesem Ort - in dem die beweglichen Räume der Strassenhändler in feste und klar abgegrenzte Räume umgeschrieben werden (s.u.) - stellen die (ehemaligen) Strassenhändler keine Bedrohung für die Sicherheit der Passanten oder für das Funktionieren des motorisierten städtischen Verkehrs dar (Dos ReIs 2003: 46; GREGÓRIO 2003:5). Dabei wird eine interessante «moralische Geographie» erzeugt: Denn im Ergebnis bedeuten die oben erwähnten politischen Massnahmen, dass die Zirkulation und Bewegung der Strassenhändler als schlechte Form der Bewegung figuriert wird, die beseitigt werden muss, um einer erwünschten und guten Form der Bewegung, eben des motorisierten Verkehrs und touristischen Flanierens, Platz zu machen.

\section{Komplexe Räumlichkeiten: feste Räume, Blasenräume und flüssige Räume}

Es ist herauszustellen, dass die Aktivitäten der Strassenhändler einen besonderen Raum hervorbringen, der anderen Mustern der Ordnung folgt, als sie etwa in der Rationalität der risky city ausbuchstabiert sind. Die potenzielle Fähigkeit des Strassenhandels, spontan und verdichtet an bestimmten Orten aufzutauchen, schafft flexible metrisch-relationale Räume, die jenen Vorstellungen ein Dorn im Auge sind, die auf feste und geordnete räumliche Strukturen ausgerich- 
tet sind. Genau diese Verdichtungen, die aus der temporären Inbesitznahme einer konkreten Stelle durch die Strassenhändler resultieren, will CosTA (1989) mit dem Begriff des Blasenraumes einfangen. Aguiar und Weber (2009) schreiben in diesem Zusammenhang passend über eine Architektur der «flüchtigen» Bewegung. Im Gegensatz zur geplanten, abgeschlossenen und damit festen Architektur der camelôdromos verweisen die Verkaufsräume der Strassenhändler auf wiederkehrende Improvisation und prinzipielle Unabgeschlossenheit.

Während mit dem Blasenraum eher das aktuelle und verdichtete Auftreten des einzelnen Strassenhändlers oder mehrerer Strassenhändler beschrieben wird, lässt sich das gesamte soziale System und die Organisation des Strassenhandels hingegen als flüssiger Raum beschreiben (Law 2002; LaW \& Mol 2001; MoL \& LaW 1994). Das Konzept des flüssigen Raumes, das explizit nicht auf einen metrischen Raum reduziert werden kann, hebt die Bedeutung von Verwandlung und Bewegung im Gegensatz zu dauerhafter Stasis und Stabilität hervor. Daran anschliessend verweist «Bewegung» auf mehr als die wahrnehmbare räumliche Bewegung des Strassenhandels und den dadurch gebildeten metrisch-relationalen Raum. Überdies ist eine interne Bewegung der Erneuerung angedeutet, die sich einer metrisch-räumlichen Wahrnehmung und Beschreibung entzieht.

In dieser Bewegung des flüssigen Raumes werden Elemente fortlaufend verändert und neu konfiguriert. Daher wird in einem flüssigen Raum keine feste Struktur hinsichtlich der Beziehungen seiner Elemente bevorzugt. Dies erlaubt es, etwa bei Störungen oder Irritationen, existierende Elemente immer wieder in neue Beziehungen zu bringen. Der Verlust von eingespielten Standorten, Unterstell- oder Lagerplätzen, das Wegfallen altbekannter Kollegen, Kunden, Lieferanten oder das Hinzukommen neuer Konkurrenten (als beispielhafte Elemente des flüssigen Raumes «Strassenhandel») führen nicht zu jähen Brüchen oder zum Kollaps. Der konkrete Strassenhändler ist damit Teil eines Systems, das sich die Fähigkeit bewahrt, auf neue Situationen durch rasche Reorganisation seiner Elemente reagieren zu können. Gerade dadurch, dass die Strassenhändler ihre Standorte und Warensortimente je nach Kundennachfrage immer wieder verändern können, wohnt dem Strassenhandel ein Moment kontinuierlicher Bewegung und fortwährender Unabgeschlossenheit inne.

Diese Fähigkeit zu fliessender und kontinuierlicher Veränderung fehlt in einem camelôdromo. Denn dort sind akute Veränderungen im Verhältnis von Ware, Ort und Kunde nahezu ausgeschlossen. Der Händler kann seinen Standort nicht verlassen und somit nicht varia- bel auf sich verändernde Nachfrageströme und -orte reagieren. Ebenso ist, aufgrund der Spezialisierung des Warensortiments, eine jähe und auf Änderungen der Situationen reagierende Neuausrichtung des Angebotes unmöglich. Eine solche Neuausrichtung würde nur abrupt stattfinden können und somit gegen eine wesentliche Bedingung des flüssigen Raumes verstossen: dass sich die Beziehungen zwischen seinen Elementen eben nur Stück für Stück ändern können (LAw 2002: 99).

Jene Bewegungen, die durch stückweise interne Veränderungen entstehen, sind nur schwerlich von aussen zu beobachten, weil die jeweilige Veränderung, bezogen auf die Gesamtheit, häufig minimal ist. Erst wenn diese Bewegungen auch einen Ortswechsel umgreifen und sich somit in einem metrischen Raum vollziehen, werden sie deutlich sichtbar und erscheinen als Blasenraum.

Es sind genau diese multiplen, metrisch-räumlichen wie nicht-metrisch-räumlichen Bewegungen, die in der Rationalität der risky city als Unordnung des Strassenhandels apostrophiert und als Quelle von Unsicherheit gelesen werden. Aus Sicht des Strassenhandels kann in ihnen jedoch auch eine besondere Ordnung erkannt werden: eine Ordnung, die auf dynamischer Stabilität beruht und eine Sicherheit des Strassenhandels konstituiert (Aguiar \& Weber 2009). Bewegungen als zentrale Facette der sozialen Organisation des Strassenhandels werden von zahlreichen Autoren hervorgehoben. Die Anthropologin PinheIro-Machado (2006) setzt sie in Analogie zu familiären Strukturen. Sie spricht symbolisch von einer Familie (uma família da rua), die für die Strassenhändler eine bedeutende Form der Verlässlichkeit und Sicherheit darstellt sowie zentral für den kommerziellen Erfolg der Händler ist. Ähnlich familiärer Strukturen basiert der Strassenhandel auf lockeren Absprachen und auf einen durch das Prinzip der vertrauenden Gegenseitigkeit ermöglichten Fluss von Informationen. Der Strassenhandel ist genau deswegen unserer Auffassung nach weder beliebig noch ungeordnet.

Nun wird deutlich, dass die Idee, den Strassenhandel in festen und singulären Standorten dauerhaft zu fixieren, seiner ihm innewohnenden - metrisch-räumlichen wie nicht-metrisch-räumlichen - Bewegung widerspricht (zu den dabei enaktivierten unterschiedlichen Räumlichkeiten vgl. Tab. 1). Die Strassenhändler finden gerade im Prinzip der Bewegung ihre Robustheit und Konstanz. Dies kennzeichnet die spezifische Sicherheit des Strassenhandels. Diese «Sicherheit des Strassenhandels» steht dann im Widerspruch zu jenen Praktiken, die über metrisch-räumliche Fixierung und Ordnung (siehe etwa die camelôdromos) eine Reduktion des Unsicheren erzielen wollen, um damit ihre spezifische Sicherheit zu gewinnen. 


\begin{tabular}{|l|l|l|}
\hline \multicolumn{1}{|c|}{$\begin{array}{c}\text { fester Raum/ } \\
\text { Containerraum }\end{array}$} & \multicolumn{1}{|c|}{ Blasenraum } & \multicolumn{1}{c|}{ flüssiger Raum } \\
\hline metrische Räumlichkeit & metrische Räumlichkeit & nicht-metrische Räumlichkeit \\
\hline unbeweglich & beweglich & beweglich \\
\hline Objekte beinhaltend & $\begin{array}{l}\text { Objekte verknüpfend } \\
\text { (relational) }\end{array}$ & $\begin{array}{l}\text { Objekt wird permanent } \\
\text { moduliert und variiert }\end{array}$ \\
\hline dauerhaft klar begrenzt & temporär klar begrenzt & $\begin{array}{l}\text { unklare, «wandernde» } \\
\text { Grenzen }\end{array}$ \\
\hline $\begin{array}{l}\text { Ordnung des Innen/ } \\
\text { Außen, Ordnung der } \\
\text { Homogenität }\end{array}$ & $\begin{array}{l}\text { Ordnung zyklischer } \\
\text { Integration und } \\
\text { Desintegration }\end{array}$ & $\begin{array}{l}\text { Ordnung der Wandelbarkeit } \\
\text { und gradueller Veränderung }\end{array}$ \\
\hline dauerhafte (feste) Einheit & $\begin{array}{l}\text { temporäres Arrangement } \\
\text { von festen Beziehungen }\end{array}$ & $\begin{array}{l}\text { dynamisch stabil, daher auch } \\
\text { kontingent und «unklar» }\end{array}$ \\
\hline
\end{tabular}

Tab. 1: Merkmale unterschiedlicher Räumlichkeiten: fester Raum, Blasenraum, flüssiger Raum Characteristics of different spaces: solid space, bubble space and fluid space Caractéristiques de différentes spatialités: espace fixe, espace-bulle, espace fluide Eigener Entwurf nach Mol \& LaW 1994 und Law 2002

Sich in Form eines flüssigen Raumes zu organisieren und als soziale Entität zu reproduzieren, gehört somit zu den kulturellen Mechanismen des Strassenhandels. Seine Sicherheiten, etwa in Form sozialer Netzwerke und flexibler Einkommensgenerierung, beruhen auf der zentralen Position einer nicht-metrischen Räumlichkeit, die Bewegung und Veränderung betont. Diese tiefliegenden Muster zur Generierung sozialer Sicherheit werden durch eine Politik der ordnenden Trennung in ihren Fundamenten angegriffen, was entsprechenden Widerstand hervorruft: In Porto Alegre kann man etwa studieren, wie sich diese flüssige Räumlichkeit immer wieder den Versuchen der Containerisierung und Einhegung entzieht, weil letztere nicht in der Lage sind, eine entsprechende Kontinuität sozialer und ökonomischer Sicherheit, wie es der mobile Strassenhandel bietet, bereit zu stellen. Es verwundert daher nicht, wenn sich Strassenhändler von den camelôdromos abwenden und sich wieder Strassen und Plätze aneignen (Aguiar \& Weber 2009).

\section{Fazit}

Die Bewegung und Wandelbarkeit des Strassenhandels bildet einen flüssigen Raum. Bewegung und Wandelbarkeit bilden die Sicherheit des Strassenhandels. Diese Konstellation ist konträr zu jenen Praktiken, die urbane Sicherheit durch eine Raumstrategie der ordnenden Trennung und Adressierung (der einzelne Strassenhändler ist nun in seiner Box im camelôdromo $\mathrm{zu}$ finden) verwirklicht sehen. Camelôdromos sind Ausdruck eines Versuchs, einen eigenständigen Sachverhalt zwar in seiner Eigenständigkeit zu akzeptieren, ihn aber zugleich «den Imperativen eines anderen Systems zu unterwerfen» (STICHWEH 2003: 95).

Es geht in den Bemühungen um die Einhegung des Strassenhandels folglich nicht darum, den Strassenhandel an sich bzw. den Aspekt seiner Informalität in Gänze zu unterbinden. Denn nach wie vor ist dieser unerlässlich, um die Bedürfnisse der Touristen und Stadtbewohner zu befriedigen oder um marginalisierten Personen Arbeit zu geben. Es geht vielmehr um Zähmung und Ordnung. Der Strassenhandel soll seine Funktion in anderen Räumen ausüben. Daher ist das camelôdromo auch so konzipiert, die wirtschaftlichen Effekte des informellen Handels zu fördern, allerdings um den Preis, diesem eine feste räumliche Form zu geben, die seiner inhärenten Ordnung räumlicher wie nicht-räumlicher Bewegungen widerspricht. Doch gerade diese Bewegungen haben zentrale Bedeutung für die soziale Sicherheit der Strassenhändler.

Die beobachtbaren Formen der Einhegung des Strassenhandels in feste (Container-) Räume können dann auch als politische Praktiken verstanden werden, die nicht nur der historisch gewachsenen Kultur des 
Strassenhandelns entgegengesetzt sind, sondern sie in ihren Grundlogiken zerstören können. Daher wird auch verständlich, dass die camelôdromos längst nicht jenen Erfolg haben, den man ihnen offiziell prognostizierte.

Insofern gilt es, solange Stadtentwicklung mehr sein soll als Ausdruck eines hegemonialen Projektes privilegierter Akteure und Institutionen, nicht allein eine Sensibilität dafür zu entwickeln, dass die Entscheidungen im Beobachtungsraster Sicherheit/Unsicherheit einer irreduziblen Kontingenz unterliegen. Es gilt überdies anzuerkennen, dass die Bestimmung dessen, was Sicherheit und Unsicherheit ausmacht, mit sehr unterschiedlichen, oftmals widersprüchlichen Räumlichkeiten einhergeht. Die multiplen Räumlichkeiten des Sozialen können allein mittels metrischer Raumbegriffe (sei es als relationaler Raum materieller Artefakte oder als fester Containerraum) nicht angemessen erfasst werden. Mit dem hier eingeführten Konzept des flüssigen Raumes soll gerade auf jene Multiplizität, Gleichzeitigkeit und auch teilweise Widersprüchlichkeit unterschiedlicher Räumlichkeiten heutiger Gesellschaften hingewiesen werden, ohne damit zu postulieren, dass sich flüssige Räume «besser» zur Darstellung aktueller gesellschaftlicher Entwicklungen eignen. Sie sind nur eine Form der Darstellung von Räumlichkeit neben anderen Formen. Aus sozialgeographischer Sicht erscheint es daher vielversprechend, diesen unterschiedlichen Räumlichkeiten und den mit ihnen verbundenen Normativitäten und Ordnungsweisen mehr Aufmerksamkeit zu schenken - auch wenn der Preis, nämlich die Akzeptanz von sich verflüssigenden raumbezogenen Vorstellungen über Sicherheit und Unsicherheit, hoch erscheint.

\section{Literatur}

Aguiar, J. \& M. Weber (2009): Por uma cidade que se move e se comunica: corpo, rua e improviso. - In: Em Questão 15,1:141-156.

Associação Viva o Centro (1994): Camelôs. Subsídios para o equacionamento do problema do comércio informal de rua e sua solução. - Documento final do workshop «O Comércio Informal de Rua e a Requalificação do Centro de São Paulo», http://www.vivaocentro.org.br 15.05.2009.

BAlzACQ, T. (2005): The three faces of securitization: political agency, audience and context. - In: European Journal of International Relations 11, 2: 171-201.

BECK, U. (1986): Risikogesellschaft. Auf dem Weg in eine andere Moderne. - Frankfurt am Main: Suhrkamp.

BROMLEY, R. (2000): Street vending and public policy: a global review. - In: International Journal of Sociology and Social Policy 20,1/2:1-29.

BuzAN, B. \& O. WÆVER (2009): Macrosecuritisation and security constellations: reconsidering scale in securitisation theory. - In: Review of International Studies 35, 2: 253-276.

BuzAN, B., WÆVER, O. \& J. DE WILDE (1998): Security: a new framework for analysis. - Boulder (Colorado): Lynne Rienner Publishers.

CARdoso, A.L.C. \& A.M.M. Martins (2005): Mercados populares. Trajetórias na apropriação de prácticas informais. - In: Colóquio Internacional Comércio, Cultura e Políticas Públicas em Tempos de Globalização, Rio de Janeiro: 1-11 (unveröffentlichtes Manuskript). Costa, E. (1989): Anel, cordão, perfume barato, uma leitura do espaço do comércio ambulante na cidade de São Paulo. - São Paulo: Editora da Universidade de São Paulo.

DonaVAN, M.G. (2008): Informal cities and the contestation of public space: the case of Bogotá's street vendors, 1988-2003. - In: Urban Studies 45, 1:29-51.

Dos ReIs, A. (2003): Questões educativas e questões no prozesso de re-estruturação urbana. $\mathrm{O}$ caso do camelódromo Dois de Julho em Jazueira-Bahia. - Chicoutimi: Editeur de l'Université du Québec à Chicoutimi.

Dos SAntos, A. (2001): Der städtische informelle Sektor in Brasilien. Das Fallbeispiel Rio de Janeiro. - Oldenburg: Elektronische Ressource, Bibliotheksund Informationssystem der Carl von Ossietzky Universität Oldenburg.

Gregório, D.A.G. (2003): Camelôs, camelódromo e informalidade: um estudo sobre o trabalho informal no comércio de Presidente Prudente. - In: Intertem@s, Vol. 5, No 5: o.S.

HAesbaert, R. (2004): O mito da desterritorialisação. Do «fim dos territórios» à multiterritorialidade. - Rio de Janeiro: Bertrand Brasil.

ITIKAWA, L. (2004): Geometrias da clandestinidade. O trabalho informal no centro de São Paulo. - In: Comin, A. \& N. Someck (Hrsg.): Caminhos para o centro. Estratégias de desenvolvimento para o região central de São Paulo. - São Paulo: Editora da UNESP: 340362.

JANUZZI, D. (2005): O desenvolvimento de Londrina e as transformações nos espaços públicos da região central. - In: Semina: Ciência Sociais e Humanas 26, Londrina: 87-94.

LAW, J. \& A. Mol (2001): Situating technoscience: an inquiry into spatialities. - In: Environment and Planning D: Society and Space 19, 5: 609-621.

LAW, J. (2002): Objects and spaces. - In: Theory, Culture and Society 19, 5/6: 91-105.

Luhmann, N. (2003): Soziologie des Risikos. - Nachdruck der Ausgabe von 1991, Berlin, New York: de Gruyter.

Meucci Nique, W. (2006): Percepção do Porto-Alegrense sobre o centro de Porto Algre. - http://www. ea.ufrgs.br/graduacao/disciplinas/adm01163/dowload/ relatorio_centro.pdf 20.09.2009. 
Middleton, A. (2003): Informal traders and planners in the regeneration of historic city centres. The case of Quito, Ecuador. - In: Progress in Planning 59, 2: 71123.

MoL, A. \& J. LAW (1994): Regions, networks and fluids: anaemia and social topology. - In: Social Studies of Science 24, 4: 641-671.

Osborne, T. \& N. Rose (1999): Governing cities. Notes on the spatialisation of virtue. - In: Environment and Planning D: Society and Space 17, 6: 737-760.

Pereira, V.S. (2007): Comércio informal, segregação sócio-especial e memória urbana: repensando as temporalidades do espaçõ urbano em São Paulo na década de 90. - http://snh2007.anpuh.org/resources/content/ anais/Veronica\%20Sales\%20Pereira.pdf 20.09.2009.

Pinheiro-Machado, R. (2006): «Tudo isso é família da gente»: relações de parantesco entre camelôs e sacoleiros em contextos locais e trans-locais. - In: Revista Anthropológicas 17, 22: 65-94.

Rose, N. (1999): Powers of freedom. Reframing political thought. - Cambridge: Cambridge University Press.

Sibley, D. (1988): Survey 13: purification of space. - In: Environment and Planning D: Society and Space 6, 4: 409-421.

SOUZA, M.J. LoPES DE (1995): O território: sobre espaço e poder, autonomia e desenvolvimento. - In: CASTRo, I.E. DE (Hrsg.): Geografia: conceitos e temas. - Rio de Janeiro: Bertrand Brasil: 77-116.

StichweH, R. (2003): Raum und moderne Gesellschaft. Aspekte der sozialen Kontrolle des Raumes. - In: Krämer-Badoni, T. \& K. Kuhm (Hrsg.): Die Gesellschaft und ihr Raum. Raum als Gegenstand der Soziologie. - Opladen: Leske \& Budrich: 93-102.

StRITZEL, H. (2007): Towards a theory of securitization: Copenhagen and beyond. - In: European Journal of International Relations 13, 3:357-383.

\section{Zusammenfassung: Verflüssigende (Un-) Sicherhei- ten. Über Räumlichkeiten des Strassenhandels am Beispiel Brasiliens}

Der Artikel untersucht die Bedeutung unterschiedlicher Räumlichkeiten im Zuge der Unterscheidung von Sicherheit und Unsicherheit. Am Beispiel des Strassenhandels in brasilianischen Städten wird zunächst gezeigt, wie dieser seitens stadtentwicklungspolitischer Akteure als ein Element der Unsicherheit gerahmt wird. Es wird ferner dargelegt, dass sich einige Massnahmen zur Minimierung dieser Unsicherheit einer raumbezogenen Strategie der Einhegung in feste Containerräume bedienen. Dies steht jedoch im Widerspruch zu den spezifischen Räumlichkeiten des Strassenhandels. So stellt die von der Politik als unsicher bewertete metrisch-räumliche und nicht-metrischräumliche Bewegung des Strassenhandels gerade für die einzelnen Strassenhändler ein Element sozialer
Sicherheit dar. Wir handeln die metrisch-räumliche Bewegung des Strassenhandels mit dem Begriff des Blasenraumes (Costa 1989) und die nicht-metrischräumliche Bewegung mit dem Konzept des flüssigen Raumes (Mol \& LaW 1994) ab. Wir betonen, dass es bei der Herstellung sozialer Ordnung wichtig ist, die spezifischen Räumlichkeiten, die in sozialen Systemen ausgebildet werden, im Blick zu behalten.

Schlüsselwörter: Strassenhandel, Sicherheit, Unsicherheit, Blasenraum, flüssiger Raum

\section{Abstract: Fluid (in)security. Spatiality of street traders in Brazil}

This article analyses the significance of different spatialities in relation to the counterparts security/insecurity. Focussing on street trading in Brazilian cities, the article describes the degree to which street traders are seen as insecure elements within contemporary discourses on urban development. This is followed by a discussion of the arrangements that have been introduced to minimise the perceived insecurity. The arrangements introduced use spatial strategies to displace street traders and contain them in fixed spaces. Yet the spatiality of these strategies is inconsistent with the intrinsically idiosyncratic spatiality of the street traders, which is a spatiality of flow and movement. While official policies deem the metric and non-metric flows and movements of street trading as threats to urban security, these same flows and movements are central to the social and economic security of the individual street trader. In order to describe the different spatialities of street trading more accurately, use is made here of the concept of bubble space (COSTA 1989, «espaços de bolha») to refer to the metric spatiality of street trading and fluid space (MoL \& LAW 1994) to refer to its non-metric spatiality. It is concluded that processes of social ordering should be sensitive to the particular spatialities that have evolved in corresponding social systems.

Keywords: street trading, security, insecurity, bubble space, fluid space

\section{Résumé: (In)sécurité fluide. La spatialité des commerces de rue brésiliens}

Cet article analyse la signification de différentes spatialités en s'appuyant sur la distinction entre sécurité et insécurité. L'exemple des villes brésiliennes permet tout d'abord de montrer que les vendeurs de rue sont appréhendés comme des éléments d'insécurité dans les discours contemporains sur le développement urbain. L'article discute ensuite les mesures mises en place pour minimiser cette insécurité et qui se basent sur un déplacement ou sur une assignation des vendeurs à certains espaces. Il montre que la spatialité de 
ces stratégies n'est pas cohérente avec celle des vendeurs de rue, fondée sur le flux et le mouvement. Alors que les politiques officielles considèrent les flux du commerce de rue comme une menace pour la sécurité urbaine, ces flux sont centraux pour la sécurité sociale et économique des vendeurs de rue. Afin de traiter des différents types de spatialité du commerce de rue de manière plus précise, l'article introduit le concept d'espace-bulle (Costa 1989, «espaços de bolha»), qui se réfère à la spatialité métrique du commerce de rue, ainsi que le concept d'espace fluide (MoL \& Law 1994) qui se rapporte à sa spatialité non métrique. L'article conclut en montrant que les aménagements urbains devraient être plus sensibles aux différentes spatialités qui se sont développées dans les systèmes sociaux.

Mots-clés: commerce de rue, sécurité, insécurité, espace-bulle, espace fluide

Dr. Marc Redepenning, Institut für Geographie, Lehrstuhl für Sozialgeographie, Friedrich-Schiller-Universität Jena, Löbdergraben 32, D-07743 Jena, Deutschland.

e-mail: marc.redepenning@uni-jena.de

Dipl.-Geogr. Henriette Neef, Universität Leipzig, Graduiertenkolleg «Bruchzonen der Globalisierung», Emil-Fuchs-Strasse 1, D-04105 Leipzig, Deutschland. e-mail: hneef@uni-leipzig.de

Prof. Dr. Edvânia Torres Aguiar Gomes, Universidade Federal de Pernambuco, Centro de Filosofia e Ciências Humanas, Departamento de Ciências Geográficas, Avenida Moraes Rego, s/n, Cidade Universitária, Cep: 50.670-901, Recife/Pernambuco, Brasilien.

e-mail: torres@ufpe.br

\section{Manuskripteingang/received/manuscrit reçu le} 23.1.2010

Annahme zum Druck/accepted for publication/accepté pour publication: 7.9 .2010 